Review

\title{
Prosocial behaviour in animals: the influence of social relationships, communication and rewards
}

\author{
Katherine A. Cronin* \\ Comparative Cognitive Anthropology Group, Max Planck Institute for Psycholinguistics, Nijmegen, The Netherlands
}

\section{A R T I C L E I N F O}

\section{Article history:}

Received 5 March 2012

Initial acceptance 10 April 2012

Final acceptance 27 July 2012

Available online 23 September 2012

MS. number: 12-00172R

\section{Keywords:}

communication

comparative psychology

dominance

helping

inequity

prosocial

proximate influence

social relationship
Researchers have struggled to obtain a clear account of the evolution of prosocial behaviour despite a great deal of recent effort. The aim of this review is to take a brief step back from addressing the question of evolutionary origins of prosocial behaviour in order to identify contextual factors that are contributing to variation in the expression of prosocial behaviour and hindering progress towards identifying phylogenetic patterns. Most available data come from the Primate Order, and the choice of contextual factors to consider was informed by theory and practice, including the nature of the relationship between the potential donor and recipient, the communicative behaviour of the recipients, and features of the prosocial task including whether rewards are visible and whether the prosocial choice creates an inequity between actors. Conclusions are drawn about the facilitating or inhibiting impact of each of these factors on the expression of prosocial behaviour, and areas for future research are highlighted. Acknowledging the impact of these contextual features on the expression of prosocial behaviours should stimulate new research into the proximate mechanisms that drive these effects, yield experimental designs that better control for potential influences on prosocial expression, and ultimately allow progress towards reconstructing the evolutionary origins of prosocial behaviour.

(C) 2012 The Association for the Study of Animal Behaviour. Published by Elsevier Ltd. All rights reserved.
Prosocial behaviour is any behaviour performed by one individual to alleviate another's need or improve their welfare. Despite much research effort, there has been a struggle to obtain a clear account of the phylogenetic emergence of prosociality (Silk 2008). This may in part be because of the variation in circumstances that surround prosocial opportunities, as it is becoming increasingly evident that prosocial expression is largely affected by context (Cronin et al. 2010; Horner et al. 2011; Skerry et al. 2011). As will be discussed in turn, there are reasons to predict that the social relationships, behaviour of the subjects and the task design have influenced the expression of prosociality in experiments; the goal of this review is to make sense of the directionality of these influences and remobilize progress towards understanding the evolutionary origins of prosociality.

This review focuses on low-cost, simple behavioural acts characterized by one individual transferring a resource or allowing resource access to another. This scope has been chosen because paradigms that involve the provisioning of tangible resources to

\footnotetext{
* Correspondence: K. A. Cronin, Comparative Cognitive Anthropology Group, Max Planck Institute for Psycholinguistics, P.O. Box 310, 6500 AH Nijmegen, The Netherlands.

E-mail address: katherine.cronin@mpi.nl.
}

another have become widely used and are leading to the generation of hypotheses that involve the integration of numerous independently conducted experiments (e.g. Warneken \& Tomasello 2009a, b; Silk \& House 2011). Thus, there exists a potentially rich source of comparative data that is poised to advance understanding of evolutionary trends in this domain of prosociality, once contextual influences are understood. Most data are from studies of nonhuman primates; in the last 10 years there have been over 30 experimental assessments of prosociality in nonhuman primates. Therefore, this review focuses primarily on nonhuman primates with data from other taxa included when available. The study of prosocial behaviour of humans, and children specifically, has a long history of experimentation that has been reviewed elsewhere (see Hay et al. 1999; Eisenberg et al. 2006; Warneken \& Tomasello 2009b); however, an evaluation of how the contextual influences investigated here have influenced prosociality in children is included in the conclusion.

Two paradigms have been commonly used to measure prosociality. The first is the prosocial choice task (e.g. Silk et al. 2005). In this task, the potential donor (hereafter 'donor' or 'actor') is typically offered the choice between one of two options. The first option provides a reward to the donor and the potential recipient (hereafter 'recipient'), commonly referred to as the ' $1 / 1$ ' option to denote that one reward is acquired by the donor and one reward by the 
recipient. The second option provides a reward for the donor only (the ' $1 / 0$ ' option). The effort required is the same for both choices; the choices differ only by whether or not the recipient receives a reward. The proportion of trials on which donors choose the prosocial option is often compared with a control condition in which no recipient is present. All of the studies reviewed below have controlled for the possibility that the behaviour can be explained by a simple preference for the option with greater rewards. Evidence of prosociality is assumed if the donor chooses the prosocial option more often when a recipient is present than absent.

The second paradigm is the out-of-reach task, which measures instrumental helping (e.g. Warneken et al. 2007). In this task, subjects are exposed to a scenario in which an individual (the recipient) appears to be in need of an object that is out of their grasp but accessible by the subject. Subjects are tested for whether or not they retrieve the item and transfer it to the recipient. In the control condition, often the same items and individuals are present but the recipient does not demonstrate need for the object.

An analysis of contextual influences is of additional importance given that prosocial reports are often based on small effect sizes. Stevens (2010) provided a summary that made clear that characteristically small effects are being interpreted as evidence of prosociality in nonhuman primates. The point here is not to call into question whether these small effects reflect a capacity for prosociality that is of biological importance (but see Stevens 2010), but to highlight that prosociality may be difficult to measure reliably and understanding contextual influences is of utmost importance.

In sum, the aim of this review is to step back from the broader questions about the evolutionary origins of prosociality in order to examine contextual features that contribute to intraspecific and interspecific variation. The review concludes with a summary of the directionality of the impact of each of the factors; throughout the review identification of possible interactions between contextual factors and suggestions for future research to understand the mechanisms that generate these trends are integrated. Because questions about the evolutionary origins of behaviour require a comparative approach, it is paramount that researchers recognize the implications of slight differences in design that may influence findings. By taking note of these features, researchers will be better positioned to design experiments that allow careful, confident comparisons within and between species, and ultimately be able to formulate testable hypotheses about the origins of prosociality in primates, both human and nonhuman.

\section{SOCIAL RELATIONSHIP BETWEEN DONOR AND RECIPIENT}

One potential influence that has caught the attention of researchers is the impact of social relationships on prosocial behaviour. Trivers's (1971) theory of reciprocal altruism provides an ultimate, evolutionary explanation for helpful acts that occur between individuals in close relationships. At a proximate, psychological level, individuals may prefer to aid those with whom they share close social bonds (Preston \& de Waal 2002). Nonhuman primates form select, stable, close social relationships that can be identified by persistent patterns of preferential proximity, frequent affiliation and rare aggression (Silk 2002). de Waal \& Suchak (2010) argued that a simple behavioural bias to direct prosociality towards those with whom an individual shares a bond will result in a balance of favours; this account does not require complex cognition. Thus, one contextual feature that is likely to influence prosociality is the social relationship between actors.

\section{Donor and Recipient are Close Social Partners}

In one study that directly tested the hypothesis that prosociality would increase with social closeness in nonhuman primates, de Waal et al. (2008) trained capuchin monkeys, Cebus apella, to differentiate between arbitrary items ('tokens') that represented selfish and prosocial options. When the actor selected the selfish token and gave it to the experimenter, the experimenter handed a reward to the actor only; when the actor selected the prosocial token, the experimenter provided a reward to both the actor and the recipient (a symbolic version of the $1 / 1$ versus $1 / 0$ prosocial choice task). By utilizing long-term observational data, the authors investigated whether prosocial responses increased with social closeness. As predicted, capuchins chose more prosocial tokens on behalf of individuals with whom they shared closer social bonds (kin and nonkin), and prosocial choices increased as subjects gained more experience of rewarding their partners.

Recently, Horner et al. (2011) employed a similar paradigm to investigate prosociality of seven female chimpanzees, Pan troglodytes. In this study, actors were offered a bucket of prosocial $(1 / 1)$ and selfish $(1 / 0)$ tokens. The researcher placed one food reward in front of the actor and one in front of the recipient, and if the actor selected a prosocial token, the experimenter handed each chimpanzee the food in front of them. If the actor chose the selfish token, the experimenter handed the actor the food but retrieved the food that was placed in front of the recipient. Actors were tested with three partners: one with whom they shared a relationship that had high scores on affiliation measures, one neutral and one low. The authors reported a positive effect of prosociality, but found no relationship with affiliation measures. There was also no effect of kinship or dominance (see Dominance Asymmetry between Donor and Recipient, below). The sample size was small and the authors did not report on the variation in affiliation measures, so it is difficult to know whether low statistical power accounts for the null effect.

Additional information about the influence of social relationships on prosociality comes from studies of tamarins and marmosets (family Callitrichidae). Callitrichids live in family groups consisting of a single breeding pair that forms a long-term, socially monogamous bond and their offspring. The breeding pairs have strong social bonds and are frequently engaged in sexual and affiliative (huddling, grooming) behaviours. Cottontop tamarins, Saguinus oedipus, tested on the prosocial choice task with their monogamous mates were more likely to choose the prosocial option over the selfish option, but this difference only emerged in a paradigm in which tamarins had ample opportunity to experience both options (Cronin et al. 2009, 2010). When tamarins could repeatedly pull (or refrain from pulling) a single $0 / 1$ tray, they continued pulling when their mate was present to receive the reward and pulling was extinguished when their mate was absent. This pattern suggests that tamarins found it rewarding to provide benefits to their long-term mates. In a later study, Stevens (2010) compared tamarins' prosocial choices for mates and strangers. An overall prosocial effect when comparing partner present versus absent conditions was not found (replicating Cronin et al. 2009), but tamarins provided more rewards to mates than strangers, supporting the interpretation that relationship quality impacts prosociality.

A recent study by Chang et al. (2011) provides additional evidence for an influence of social relationship on prosociality. Rhesus macaques, Macaca mulatta, were tested with a familiar and a less familiar partner. The authors utilized instrumental and Pavlovian conditioning to associate the following reward scenarios with unique visual cues: juice delivered to the actor only $(1 / 0)$, the actor and the recipient $(1 / 1)$, the recipient only $(0 / 1)$ or nobody 
$(0 / 0)$. The macaques were later provided with forced-choice trials between the visual cues that had been associated with each of the reward distributions. Although there were only two donor subjects, the macaques demonstrated a preference for $(0 / 1)$ over $(0 / 0)$ for familiar recipients more so than for less familiar recipients (see also Masserman et al. 1964 in which macaques preferentially avoided delivering a negative stimulus to closer social partners).

Investigating the impact of social relationships on prosociality is a challenging endeavour requiring either a larger sample size than available to many researchers or a longitudinal approach investigating prosocial responses over the development of a relationship. Results support the theoretical predictions that prosociality is more likely between individuals who share a close social bond; however, the mechanism supporting this effect is not known and given the prevalence of null results it is clear that close social bonds are not sufficient to produce prosocial responses Pelé et al. 2009 (see also Silk et al. 2005; Vonk et al. 2008; Massen et al. 2011). The temporal patterns of prosocial responses in some studies raise the intriguing suggestion that actors experience the rewards of others when providing benefits to close social partners, providing one proximate mechanism that facilitates prosocial responses between close social partners (de Waal et al. 2008; Chang et al. 2012). To evaluate this potential mechanism further, it would be beneficial if reports included the time course of prosocial responses. If animals are experiencing rewarding properties from providing benefits to close social partners, it will be important to consider that prosocial effects may be strongest among close social partners late in experiments. Regardless, when comparing across studies it is prudent to acknowledge that closely associated individuals will have a higher probability of demonstrating prosociality than distantly associated individuals.

\section{Dominance Asymmetry between Donor and Recipient}

In many primate species, dominance hierarchies dictate priority of access to resources such as food, and subordinate individuals may concede resources to more dominant individuals to avoid aggression (Preuschoft \& van Schaik 2000). Therefore, if investigations of resource transfer reflect naturally occurring patterns of resource allocation, one would predict that prosociality would be more likely to occur on behalf of dominant rather than subordinate recipients. That is, one would expect benefits to be transferred 'up' the hierarchy.

Yamamoto et al. (2009) tested pairs of unrelated, adult female chimpanzees with known dominance relationships in side-by-side booths with desirable fruit juice located just outside. The subjects required a unique tool to access the juice in front of their own booth, but each subject's tool was located inside the partner's booth. The authors reported a prosocial effect in this study; chimpanzees transferred tools that were useful to the partner more often than tools that were not (see also Yamamoto et al. 2012). The authors also tested mother-offspring pairs; they showed a similar effect. Furthermore, subordinate individuals transferred the tool to dominant partners more often than the reverse. However, only three females were tested (in three pairwise combinations) so this effect of dominance should be considered in concert with additional studies.

An additional account of prosociality directed up the hierarchy in chimpanzees comes from Melis et al. (2011). In this study, a donor and recipient were housed across from each other; between them was a ramp with a reward positioned for delivery to the recipient's room. The reward was held back by a chain that the donor could release. The authors reported a positive effect of prosociality; however, this effect was limited to a comparison between active recipients who made loud noises (for example by banging the chain or bars) and an empty cage; chimpanzees did not provide significantly more rewards to a passive recipient than they did to an empty cage. Whether the donors were acting in order to cease the noise created by the recipient or responding prosocially to expressions of interest in the reward is unclear (see Direct Requests for Assistance, below). This study, however, did report a trend for more prosocial choices made by subordinate individuals on behalf of dominant individuals, in line with the tool transfer results reported for chimpanzees (Yamamoto et al. 2009).

Although these results suggest that chimpanzees directed prosocial responses up the hierarchy, the prosocial choice task utilizing tokens described above also argued for a positive effect of prosociality in chimpanzees but in contrast found a trend for higher ranking individuals to be more prosocial than lower ranking individuals (Horner et al. 2011). Therefore, it is currently unclear whether rank provides any predictive value for the direction of prosociality in chimpanzees.

Outside of chimpanzees, a pattern emerges that indicates prosociality is more often directed down the hierarchy. Takimoto et al. (2010) tested four capuchin monkeys, each with a dominant and subordinate recipient on a modified version of the prosocial choice task and found a prosocial effect when the actors were tested with subordinate but not dominant recipients. The same pattern was found in a study of capuchin monkeys by Lakshminarayanan \& Santos (2008) although all subjects were tested with a single, low-ranking recipient.

Prosociality directed down the hierarchy has been reported for multiple species of macaques. Dominant longtailed macaques, Macaca fascicularis, made more prosocial choices overall than did subordinate macaques tested on the prosocial choice task (Massen et al. 2010), although the relative rank distance between actors and recipients was not as important as the absolute rank position held by the actor. Among these animals, rank mattered more than relationship quality (assessed by time in contact and grooming; Massen et al. 2011). In Chang et al.'s (2011) study of vicarious reward in rhesus macaques described above, prosocial responses were more often directed from dominant towards subordinate individuals. Finally, the same directional effect of rank has been reported for macaques tested on the $1 / 1$ versus $1 / 0$ prosocial choice task (Colman et al. 1969) and on a task that measured the duration of food access permitted to another individual (Schaub 1996). In the latter report, rank was found to influence findings whereas kinship was not.

In sum, the evidence suggests that outside of chimpanzees, prosociality is more often directed down the hierarchy, with dominant individuals showing more prosocial actions on behalf of individuals subordinate to them. Some authors (e.g. Massen et al. 2010) have interpreted this effect as consistent with the honest signalling or handicap principle proposed by Zahavi (1977), which predicts dominant individuals will behave in ways that benefit others in order to advertise their dominance, but this remains to be tested. At a proximate level there may be many influences that result in prosociality occurring more often by dominant individuals. For example, dominant individuals may be more likely to approach the apparatus, they may be less anxious when near desirable resources and be better able to evaluate the choices presented, or perceive a greater chance of obtaining food for themselves once it is in the possession of a subordinate individual. To determine the proximate mechanisms that lead to prosociality being directed down the hierarchy, it is necessary for researchers to report the behaviour of subjects both before and after the resources are allocated and not simply the relative amount of prosocial and selfish choices. 


\section{THE BEHAVIOUR OF THE RECIPIENT}

In many designs, recipients are positioned near a desirable resource but unable to access it alone. Often the recipients demonstrate their motivation to obtain the resource by reaching out towards it or vocalizing (e.g. Burkart et al. 2007; Cronin et al. 2009). Prosociality may be more likely when recipients are distressed and expressing interest in the reward. Preston \& de Waal (2002) and de Waal (2008) formulated the perception action mechanism (PAM) model, which proposes that one individual (the actor) has access to the subjective state of another (the recipient) through emotional contagion characterized by activation of the actor's own neural and bodily representations. The actor, in a sense, shares the recipient's emotions and needs, which fosters behaviours that improve the emotional state and alleviate the needs of the recipient. Furthermore, this model predicts that emotional contagion will be stronger between individuals who are socially close, as these individuals will identify more easily with the state of the other. Therefore, there may be an interaction between relationship quality, discussed above, and behaviour such that signals of need increase prosociality on behalf of close social partners more so than distant ones. The following sections focus on the results of prosocial experiments that have reported the behaviour of the recipients, treating separately cases in which the recipient is expressing interest in the reward and cases in which recipients actually direct requests towards the actor, as these categories of behaviour may differ in their effectiveness at eliciting prosociality (e.g. Warneken et al. 2007).

\section{Recipient Expresses Interest in the Reward}

Chimpanzees and capuchin monkeys have been tested in the out-of-reach paradigm with a human experimenter as the recipient (Warneken et al. 2007; Barnes et al. 2008). In these studies, both species helped on low (but not high) effort versions of this task more often when the human reached out for the reward compared to when they did not. The chimpanzees became less responsive to the reaching signal over the course of the experiment, and in both species helping behaviour was maximally seen in about half of the trials. Thus reaching by human experimenters may have a moderately positive effect on prosociality in the case of nonfood rewards. However, one cannot be sure the subjects were interpreting the intraspecific communication as the experimenters intended, and it is difficult to discern how positive results translate into interspecific interactions. Therefore the following discussion focuses on studies that investigated whether subjects behave prosocially towards conspecifics expressing interest in the item, rather than humans.

In one comprehensive experiment Pelé et al. (2009) investigated whether any of the four nonhuman great ape species would provide an out-of-reach token (valuable to the recipient only) to a conspecific. Only orang-utans, Pongo pygmaeus, regularly held out their hand in an open-handed begging gesture or pointed directly at tokens that were in the possession of their partner, and they were also the only species that regularly transferred tokens between partners. In this and a subsequent study (Dufour et al. 2009), subjects were more likely to receive a token from their partner if they were expressing interest via an open-handed beg or pointing at the token. However, the effectiveness of the gesturing decreased throughout the study. Reaching out towards the item did not continuously elicit a prosocial response, even from individuals who had been previously responsive to the signal.

In an early study of prosociality spanning rhesus macaques, pigtailed macaques, Macaca nemestrina, spider monkeys, Ateles geoffroyi, capuchin monkeys and hamadryas baboons, Papio hamadryas, Wolfle \& Wolfle (1939) presented subjects with the option to pull a cord to provide a reward to their partner. The authors included a 'screen' condition in which the donor could see the recipient but not whether there was a food item on the tray. The authors surmised that if the donors were attending to the behaviour of the recipients (which often included reaching), they would pull on food-present trials more often than on food-absent trials even though they could not see the food. None of the nonhuman primates pulled more often in the screen condition when food was present compared to absent, indicating that the subjects were not reacting to the behaviour of their partners. Expressing interest in the rewards may have facilitated prosocial responses in one nonprimate species tested recently on the prosocial choice task; jackdaws, Corvus monedula, made more prosocial choices when recipients approached the food compared to when they were at a distance. The authors attributed this effect to local or stimulus enhancement rather than an increased appreciation of the partner's need (Schwab et al. 2012).

Reaching towards the reward has not facilitated prosocial responding in any nonhuman primate tested in the prosocial choice task. Chimpanzees failed to choose the prosocial option when a partner was present to receive the reward compared to when a partner was absent (Silk et al. 2005; Jensen et al. 2006) and when chimpanzees reached out towards the reward, actors were no more likely to choose the prosocial option (Vonk et al. 2008). Cottontop tamarins provided fewer rewards to partners on trials during which their partner reached out for the reward compared to trials when they did not (Cronin et al. 2009). Although cooperatively breeding common marmosets did show prosociality on the prosocial choice task, reaching for the reward had no effect (Burkart et al. 2007). Burkart et al. argued that the marmosets exhibited 'unsolicited prosociality', that is, marmosets were motivated to act in the best interest of others regardless of whether the recipient demonstrated interest. Given that the natural behaviour of cooperatively breeding marmosets and tamarins involves much collaborative care and passive and active food sharing this may be possible (Snowdon \& Ziegler 2007), but prosocial responses were not near ceiling and it is unclear how a species that has evolved prosocial motivations would be immune to this behavioural indication of need. (See Jaeggi et al. 2010 for a review of naturally occurring food sharing in primates and responses to signals of need.)

If a prosocial effect is found but behavioural signals of need do not increase prosociality, it would be of interest to investigate whether there may be a more parsimonious explanation for the apparent unsolicited prosociality. One possibility is that the subjects are interpreting the situation differently from what the experimenters intended (e.g. subjects perceive they can somehow obtain the food for themselves, but when the recipient reaches for the reward donors deem it less likely that they will obtain it for themselves, and reduce their 'prosocial' actions). A second possibility is that subjects possess some prosocial motivations but that the behaviour of the recipient is increasing the subject's own interest in the food and minimizing the attention paid to the recipient's potential benefit. The conclusion of unsolicited prosociality requires careful ruling out of other explanations.

Taken together, findings indicate that the recipients' expression of interest in rewards, often measured via reaching, does little to facilitate prosociality in nonhuman primates. In some great apes, expressions of interest in out-of-reach tasks may increase prosocial responses initially but the effect appears to wane. Why prosocial responses do not increase when recipients express interest in the rewards is not clear, although the pattern is apparent across multiple studies employing the prosocial choice task. Determining why this is the case should be an important aim moving forward, as one reason may be that the subjects are not interpreting the task as the experimenters have intended. None the less, when synthesizing 
results across multiple studies, researchers should bear in mind that the opportunity for the donor to observe the recipient's interest in the reward does not necessarily increase the likelihood of a prosocial reaction on these tasks, and may even inhibit it.

\section{Direct Requests for Assistance}

When the recipient unambiguously directs behaviour towards the actor rather than the reward, the behaviour is better described as a direct request. Direct requests have been hypothesized to increase prosociality because the goals of the recipient are more salient (e.g. Warneken et al. 2007; Yamamoto \& Tanaka 2009a). Among nonhuman primates, direct requests have only been reported for chimpanzees (with the potential exception of the orangutan behaviour reported in Pelé et al. 2009; Dufour et al. 2009, above). Yamamoto \& Tanaka (2009b) tested chimpanzees in sideby-side testing booths and provided them with tokens that, when inserted into a vending machine, produced a reward for the partner. One subject adopted the strategy of physically 'nudging' the partner, that is, reaching through a small opening in the clear divider that separated booths and pushing on the partner's shoulder. This behaviour increased token insertion by the 'nudged' animal. When chimpanzees needed tools to access juice outside their testing booth (Yamamoto et al. 2009, 2012), there was an increase in tool transfers following requests by the recipients, which took the form of reaching out towards and vocalizing at the partner. Likewise, in the study described above in which chimpanzees could release a chain to deliver food to their partner, chimpanzees released the chain more often when their partner was making a noise with the chain, interpreted by the authors as a request (Melis et al. 2011). In contrast, Horner et al. (2011) reported that the chimpanzees tested in the prosocial choice task with tokens did not increase prosocial responses following direct requests or pressure, but did not differentiate between the two.

In sum, requests that are unambiguously directed at donors rather than the rewards have been slightly more effective, but reports of direct requests have been rare and to date limited to chimpanzees. Moving forward, it will be important to distinguish between direct requests and harassment (Gilby 2006), as the motivation to transfer resources following each may differ. Noting whether direct requests occur during future experiments is of interest for at least two reasons. First, if direct requests occur, this provides some indication that the subjects involved in the study have a thorough understanding of the experimental set-up and which individual is responsible for the distribution of rewards. Second, if a lack of understanding of the goal of the recipient is impeding prosociality, as has been argued by Warneken et al. (2007; but see Yamamoto et al. 2012), direct requests are arguably the most straightforward expression of the recipients' goals. Therefore, if researchers report the lack of a prosocial effect in cases where direct requests are occurring, this would provide strong evidence against prosocial preferences.

\section{FEATURES OF THE TASK}

\section{The Presence of Desirable Food}

The following section focuses on the influence of food visibility on prosociality. Hirata (2007) argued that, at least in the case of chimpanzees, the presence of food may elicit competitive, selfish aspects of behaviour and mask the expression of prosociality. This effect is likely to differ by species depending on the amount of species-typical food competition (see also Hare et al. 2007; Wobber et al. 2010). Therefore, to determine whether the presence of food inhibits prosociality, experiments with and without visible food rewards conducted within the same species are compared here, first for chimpanzees and then for capuchin monkeys. These two species are chosen because they have been subjects of multiple prosocial experiments that have varied on this dimension and reportedly differ in species-typical feeding tolerance (de Waal et al. 2008).

To date, no chimpanzee study has demonstrated prosocial behaviour when the task involved visible food. At least three experimental attempts have been made. Both Silk et al. (2005) and Jensen et al. (2006) presented chimpanzees with trays baited with fresh fruits in the $1 / 1$ and $1 / 0$ reward distributions and chimpanzees were not prosocial. Likewise, in a study by Brosnan et al. (2009) designed to investigate whether reciprocity would facilitate prosocial responses, chimpanzees could act prosocially and transfer chocolate bars to conspecifics and again there was no prosocial effect.

In contrast, designs that conceal or distance food rewards from chimpanzees have produced some positive results. Warneken et al. (2007) trained subjects to pull out a pin attached to a chain that was securing a door shut. Once the pin was removed, the chain was relaxed and the door could be opened that allowed a recipient to access food. The donor could see the recipient but not the food, and chimpanzees behaved prosocially, presumably responding to the behaviour of the recipient. Additionally, small positive effects were obtained with chimpanzees in other studies when the actor's access to the food was blocked by multiple barriers (Melis et al. 2011), when the food rewards were concealed in wrapping (Horner et al. 2011), and when the recipient's reward was not visible while the subject made choices (Yamamoto et al. 2009, 2012).

Therefore, food visibility is one factor that appears to decrease the likelihood of prosociality by chimpanzees in captive settings. However, simply concealing food is not sufficient to elicit prosocial reactions in chimpanzees; in three experiments reported by Pelé et al. (2009) utilizing token exchange, subjects did not spontaneously transfer tokens to partners who could then exchange the tokens for food rewards. Similarly, Vonk et al. (2008) concealed the food in the prosocial choice task in shredded paper inside a plastic ball and a prosocial effect was not found.

In contrast, capuchin monkeys typically show more tolerance and less competition over food (de Waal et al. 2008), so the presence of food in the environment of the actor may not be as detrimental to capuchin prosociality. Unlike chimpanzees, evidence for prosociality has emerged from methodologies in which food is visible to the actors during decision making. Lakshminarayanan \& Santos (2008) presented capuchins with a version of the prosocial choice task in which one tray was baited with a high-value food reward for both actors and the other tray was baited with a high-value food reward for the donor and a lower value food reward for the recipient (high/high versus high/ low), and capuchins chose the high/high tray more often when a partner was present than absent. At least two other prosocial choice tasks with capuchins involving visible food also yielded positive results (Brosnan et al. 2010; Takimoto et al. 2010). From these studies and from studies demonstrating prosociality when food rewards are not a salient design feature (e.g. Westergaard et al. 2007; Barnes et al. 2008), it seems that prosociality by capuchins is not inhibited by the presence of desirable food items as it is in chimpanzees.

In sum, the presence of visible, desirable food may be an inhibiting influence on prosocial expression in nonhuman primates but this effect may differ between species with different levels of tolerance around food. This interaction is supported by results from the two species examined above, but until more data are available on prosociality in different contexts the relationship remains 
speculative. In rats, Rattus norvegicus, prosociality has been reported in the form of releasing a trapped cagemate (Rice \& Gainer 1962), and has persisted in conditions in which food was present and would have to be shared with the liberated individual (Ben-Ami Bartal et al. 2011). Exactly how competition and tolerance around desirable resources impacts the expression of prosociality within and between species promises to be a fruitful realm of future study.

\section{The Prosocial Choice Generates Inequity}

There may be a tipping point between options that are prosocial and equitable, and options that are prosocial but create inequity between the donor and recipient. Disadvantageous inequity, which is generated when another individual receives greater benefits than oneself, is aversive and will be avoided or corrected if possible (Brosnan \& de Waal 2003; Fehr \& Fischbacher 2003; but see Roma et al. 2006). The avoidance of options that generate disadvantageous inequity may compete with prosocial preferences if and when they do exist, if the motivation to be prosocial is less than the motivation to avoid inequity.

As reviewed above, capuchin monkeys have demonstrated prosocial behaviour on multiple prosocial choice tasks. However, Fletcher (2008) reported that capuchin monkeys presented with a $1 / 1$ versus $1 / 3$ choice task preferred $1 / 1$ over $1 / 3$ in the presence of a partner. Taken together, these findings indicate that the motivation for the equitable distribution in capuchin monkeys may override prosocial motivations, as the prosocial response would have been to choose $1 / 3$ over $1 / 1$. Two additional studies addressed this directly by integrating conditions with and without inequity, and reported that prosocial responses of capuchin monkeys are dampened when the reward for the recipient is valued more than the reward for the donor (de Waal et al. 2008; Brosnan et al. 2010). However, inequity aversion is not strong enough to override prosociality under all circumstances, as there is evidence for prosociality when the donor receives no reward or a lower quality reward than the recipient (Lakshminarayanan \& Santos 2008; de Waal et al. 2008), at least among close social partners (Donor and Recipient are Close Social Partners, above; Brosnan et al. 2005). Furthermore, there appears to be little difference in results between the $1 / 1$ versus $1 / 0$ and $0 / 0$ versus $0 / 1$ designs (Stevens 2010). This balance between prosociality and inequity aversion may be important to consider when interpreting other results as well. Studies that have failed to provide evidence in support of prosocial preferences may have been affected by an aversion to disadvantageous inequity. In future experiments, titration of reward values may elucidate whether inequity aversion is competing with prosociality and some caution should be taken when comparing studies in which the reward differential between donor and recipient was not constant.

\section{CONCLUSION}

A lack of analysis of contextual influences on prosociality has hampered our ability to identify evolutionary patterns of prosociality. This lack of attention may in part be because of the recent resurgence of interest in identifying the evolutionary origins of prosociality; the eagerness has led to the quick generation of hypotheses that rest largely on null results, small effects, and findings that are often contradicted by subsequent reports. This review has attempted to capitalize on this recent influx of data by scrutinizing how slight differences in the context in which prosociality was assessed may have impacted behaviour.

The evidence for the directional of influences of some factors assessed in this review is summarized in Table 1. Unlike the preceding discussion that has made use of comparisons across studies, the table includes only reports that have assessed the factor of interest within a single study. Considering all available information, in general, prosocial responses are more often reported on behalf of close social partners. Furthermore, it seems that prosociality is more often directed from dominant towards subordinate individuals. There are many potential proximate and ultimate accounts for this trend, and it is not yet clear what is driving this effect. Notably, this pattern is not stable among chimpanzees, perhaps because more results are available for chimpanzees or because chimpanzees are influenced by other factors to a greater extent than some other primates. None the less, dominance influenced findings among most species studied, and researchers should avoid generalizations about species' differences in prosociality based on reports that have assessed prosociality between subjects of different rank relations.

One of the most curious trends to emerge from this review is that prosociality is less likely when recipients exhibit interest in the reward. This pattern is counterintuitive and begs additional investigation. Researchers should be cautious of this effect and strongly question whether a significant prosocial effect that emerges from this situation is reflective of prosocial intentions by the subjects. Providing additional behavioural data both before and after the prosocial actions will help interpretation of this trend, as well as the dominance effect.

In contrast, when recipients direct attention or requests at the donors, prosociality was more likely. However, reports of behaviour directed towards the donor were rare, expressed only by some chimpanzees and possibly orang-utans thus far. Whether this reflects a cognitive difference between monkeys and the great apes such as limited theory of mind capabilities and/or the lack of a thorough understanding of how the experimental set-up works is unknown. The reporting of donor-directed behaviour when it does occur would help elucidate what the subjects understand about the set-up and whether donors are responsive to or dismissive of behavioural signals that indicate an opportunity for prosocial action. Again, incorporating more behavioural analyses into studies of prosociality is key to assessing prosocial motivations of the subjects, and whether similar prosocial outcomes have been spurred by different proximate mechanisms.

Although this review focused on prosocial experiments that involved the transfer of a resource from one individual to another, many variants in methodology remain. Primates showed fewer prosocial responses when the act would result in a large, disadvantageous inequity for the donor, and there was an inhibiting influence on prosociality when benefits were visible to the donor. Importantly, this effect differed across species, and the inhibiting influence of visible food may be stronger in species with characteristically high levels of food competition.

Integration of these findings with the developmental literature allows an assessment of whether humans present a distinct primate case or demonstrate prosocial behaviour that would be predicted from our nonhuman primate relatives. Among children, prosocial responses are generally facilitated when the recipient is a friend, and inhibited when the recipient is a known individual who is not a friend (Costin \& Jones 1992; Fehr et al. 2008; Olson \& Spelke 2008; Moore 2009; but see Berndt 1981), demonstrating a facilitating effect of relationship quality as found in many nonhuman primates. There is some evidence that inequity aversion can compete with prosocial motivations in children (e.g. Fehr et al. 2008) but as is the case for nonhuman primates, the degree of inequity that is tolerated and at what point children experience a conflict between prosocial motivations and inequity aversion is not yet clear. Unlike nonhuman primates however, children show a reliable increase in prosocial responses when the recipient expresses interest in the benefits (Warneken \& Tomasello 2006, 
Table 1

Directional effects of potential factors influencing prosociality

\begin{tabular}{|c|c|c|c|}
\hline \begin{tabular}{|l|} 
Factor \\
\end{tabular} & $\begin{array}{l}\text { Studies reporting that the factor } \\
\text { is associated with increased } \\
\text { prosociality }\end{array}$ & $\begin{array}{l}\text { Studies examining the factor and } \\
\text { finding no effect on prosociality }\end{array}$ & $\begin{array}{l}\text { Studies reporting that the factor } \\
\text { is associated with decreased } \\
\text { prosociality }\end{array}$ \\
\hline \multirow[t]{4}{*}{$\begin{array}{l}\text { Close social relationship between } \\
\text { donor and recipient }\end{array}$} & $\begin{array}{l}\text { Rhesus macaques, Macaca mulatta, } 15 \\
\text { Other (antisocial choice task) } \\
\text { Masserman et al. } 1964\end{array}$ & $\begin{array}{l}\text { Long-tailed macaques, Macaca fuscicularis, } 20 \\
\text { Prosocial choice task } \\
\text { Massen et al. } 2011\end{array}$ & \\
\hline & $\begin{array}{l}\text { Capuchins, Cebus apella, } 8 \\
\text { Prosocial choice task } \\
\text { de Waal et al. } 2008\end{array}$ & $\begin{array}{l}\text { Chimpanzees, Pan troglodytes, } 7 \\
\text { Prosocial choice task } \\
\text { Horner et al. } 2011\end{array}$ & \\
\hline & $\begin{array}{l}\text { Cottontop tamarins, Saguinus oedipus, } 5 \\
\text { Prosocial choice task } \\
\text { Stevens } 2010\end{array}$ & $\begin{array}{l}\text { Gorillas, Gorilla gorilla, } 3 \\
\text { Bonobos, , Pan panistus, } 5 \\
\text { Chimpanzees, Pan troglodytes, } 4 \\
\text { Orangutans, Pongo pygmaeus, } 4 \\
\text { Out-of-reach task } \\
\text { Pelé et al. } 2009\end{array}$ & \\
\hline & $\begin{array}{l}\text { Rhesus macaques, Macaca mulatta, } 2 \\
\text { Prosocial choice task } \\
\text { Chang et al. } 2011\end{array}$ & & \\
\hline \multirow[t]{4}{*}{$\begin{array}{l}\text { Donor is higher ranking than the } \\
\text { recipient }\end{array}$} & $\begin{array}{l}\text { Capuchins, Cebus apella, } 4 \\
\text { Prosocial choice task } \\
\text { Takimoto et al. } 2010^{\dagger}\end{array}$ & $\begin{array}{l}\text { Rhesus macaques, Macaca mulatta, } 15 \\
\text { Other (antisocial choice task) } \\
\text { Masserman et al. } 1964\end{array}$ & $\begin{array}{l}\text { Chimpanzees, Pan troglodytes, } 14 \\
\text { Prosocial choice task* } \\
\text { Melis et al. } 2011 \text { (trend) }\end{array}$ \\
\hline & $\begin{array}{l}\text { Long-tailed macaques, Macaca fuscicularis, } 20 \\
\text { Prosocial choice task } \\
\text { Massen et al. 2010; } 2011\end{array}$ & $\begin{array}{l}\text { Chimpanzees, Pan troglodytes, } 11 \\
\text { Prosocial choice task } \\
\text { Jensen et al. } 2006\end{array}$ & $\begin{array}{l}\text { Chimpanzees, Pan troglodytes, } 3 \\
\text { Out-of-reach task (tool transfer) } \\
\text { Yamamoto et al. } 2009\end{array}$ \\
\hline & $\begin{array}{l}\text { Rhesus macaques, Macaca mulatta, } 2 \\
\text { Prosocial choice task } \\
\text { Chang et al. } 2011\end{array}$ & $\begin{array}{l}\text { Capuchins, Cebus apella, } 8 \\
\text { Prosocial choice task } \\
\text { de Waal et al. } 2008\end{array}$ & \\
\hline & $\begin{array}{l}\text { Chimpanzees, Pan troglodytes, } 7 \\
\text { Prosocial choice task } \\
\text { Horner et al. } 2011 \text { (trend) }\end{array}$ & & \\
\hline \multirow[t]{4}{*}{$\begin{array}{l}\text { Recipient expresses interest in the } \\
\text { reward }\end{array}$} & $\begin{array}{l}\text { Orangutans, Pongo pygmaeus, } 4 \\
\text { Out-of-reach task } \\
\text { Pelé et al. } 2009\end{array}$ & $\begin{array}{l}\text { Pigtail macaques, Macaca nemestrina, } 3 \\
\text { Hamadryas baboon, Papio hamadryas, } 1 \\
\text { Rhesus macaque, Macaca mulatta, } 2 \\
\text { Prosocial choice task* } \\
\text { Wolfle \& Wolfle } 1939\end{array}$ & $\begin{array}{l}\text { Cottontop tamarins, Saguinus oedipus, } 14 \\
\text { Prosocial choice task } \\
\text { Cronin et al. } 2009\end{array}$ \\
\hline & $\begin{array}{l}\text { Jackdaws, Corvus monedula, } 7 \\
\text { Prosocial choice task } \\
\text { Schwab et al. } 2012\end{array}$ & $\begin{array}{l}\text { Common marmosets, Callithrix jacchus, } 15 \\
\text { Prosocial choice task } \\
\text { Burkart et al. } 2007\end{array}$ & $\begin{array}{l}\text { Orang-utans, Pongo pygmaeus, } 2 \\
\text { Out-of-reach task } \\
\text { Dufour et al. } 2009\end{array}$ \\
\hline & & $\begin{array}{l}\text { Chimpanzees, Pan troglodytes, } 7 \\
\text { Prosocial choice task } \\
\text { Vonk et al. } 2008\end{array}$ & $\begin{array}{l}\text { Capuchins, Cebus apella, } 4 \\
\text { Prosocial choice task } \\
\text { Takimoto et al. } 2010^{\dagger}\end{array}$ \\
\hline & & $\begin{array}{l}\text { Gorillas, Gorilla gorilla, } 3 \\
\text { Bonobos, Pan paniscus, } 5 \\
\text { Chimpanzees, Pan troglodytes, } 4 \\
\text { Out-of-reach task } \\
\text { Pelé et al. } 2009\end{array}$ & \\
\hline \multirow[t]{3}{*}{$\begin{array}{l}\text { Recipient directs requests at the } \\
\text { donor }\end{array}$} & $\begin{array}{l}\text { Chimpanzees, Pan troglodytes, } 6 \\
\text { Other (token transfer) } \\
\text { Yamamoto \& Tanaka 2009b (trend) }\end{array}$ & $\begin{array}{l}\text { Chimpanzees, Pan troglodytes, } 7 \\
\text { Prosocial choice task } \\
\text { Horner et al. 2011 }\end{array}$ & \\
\hline & $\begin{array}{l}\text { Chimpanzees, Pan troglodytes, } 9 \\
\text { Out-of-reach task (tool transfer) } \\
\text { Yamamoto et al. } 2009\end{array}$ & & \\
\hline & $\begin{array}{l}\text { Chimpanzees, Pan troglodytes, } 14 \\
\text { Prosocial choice task" } \\
\text { Melis et al. } 2011^{\mathrm{s}}\end{array}$ & & \\
\hline
\end{tabular}

Only studies that directly examined the factor and tested subjects with conspecific recipients are included. Following the species' common name is the scientific name and sample size, then the task type and reference. * Modified prosocial choice task: 0/1 versus no action.

Cannot statistically separate the potential effects of dominance and begging.

Direct requests not statistically separated from harassment behaviours.

Making noise with a chain interpreted by the authors and here as a request. 
2007; Warneken et al. 2007) or directs request at the donor (Wolfle \& Wolfle 1939; Hay et al. 1999; Svetlova et al. 2010; but see Rheingold et al. 1976). Thus there may be some important differences in how communication systems interact with prosociality in humans compared with other primates, especially considering the frequency with which communication decreases prosocial responding by nonhuman primates.

Experimental assessments of prosociality in other taxa with similar paradigms have been rare (Baron \& Littman 1961; Schwab et al. 2012) but as results become available this information will be essential to reconstructing the evolutionary origins of prosocial behaviour. It has been argued that some basic forms of otherregarding preferences have deep evolutionary roots and seemingly altruistic or prosocial behaviours have been reported under wild conditions for many species (de Waal 2008; Bekoff \& Pierce 2009). If this is so, we stand to learn much about how the social environment and other contextual features influence the expression of prosocial behaviour in the greater animal kingdom.

In sum, this review provides a comprehensive account of the directional influence of features that have often varied between studies of prosociality in nonhuman primates. The factors discussed here were chosen based on theory and available data, but the analysis is not exhaustive and other factors may be at play as well. For example, it remains to be assessed how factors that have some influence in human prosociality (Eisenberg et al. 2006) such as the age, sex or level of need of the recipient or the actor may influence prosocial responses in animals. This review has demonstrated that the context in which prosociality is studied influences prosocial expression, sometimes in predictable directions. The exciting next step is to determine the mechanisms that generate these directional effects. Experiments aimed at determining how (behaviourally, cognitively, hormonally) it comes to be that, for example, prosocial behaviour can be dampened in the case of greater communication by the recipient or heightened on behalf of subordinate individuals provide new lines of research following from the trends identified here. This review highlights a need for more detailed behavioural reporting in order to understand why these directional effects are emerging and what these patterns can tell us about prosocial motivations more generally. Finally, this synthesis should be taken into account in future attempts to synthesize research and generate hypotheses about prosocial origins.

\section{Acknowledgments}

I thank C.T. Snowdon for thoughtful discussions that contributed to the development of the ideas expressed in this review, and C.T. Snowdon, D.J. Acheson and two anonymous referees for providing valuable feedback on the manuscript. Financial support was provided in part by a Hilldale Professorship to C.T. Snowdon, by the Comparative Cognitive Anthropology Group of the Max Planck Institute for Psycholinguistics, and by the Netherlands Organization for Scientific Research.

\section{References}

Barnes, J. L., Hill, T., Langer, M., Martinez, M. \& Santos, L. R. 2008. Helping behaviour and regard for others in capuchin monkeys (Cebus apella). Biology Letters, 4, 638-640.

Baron, A. \& Littman, R. 1961. Studies of individual and paired interactional problem-solving behaviour of rats: II. solitary and social controls. Genetic Psychology Monographs, 64, 129-209.

Bekoff, M. \& Pierce, J. 2009. Wild Justice: The Moral Lives of Animals. Chicago: University of Chicago Press.

Ben-Ami Bartal, I., Decety, J. \& Mason, P. 2011. Empathy and pro-social behavior in rats. Science, 334, 1427-1430.

Berndt, T. J. 1981. Age changes and changes over time in prosocial intentions and behavior between friends. Developmental Psychology, 17, 408-416.
Brosnan, S. F. \& de Waal, F. B. M. 2003. Monkeys reject unequal pay. Nature, 425 297-299.

Brosnan, S. F., Schiff, H. C. \& de Waal, F. B. M. 2005. Tolerance for inequity may increase with social closeness in chimpanzees. Proceedings of the Royal Society $B$ 272, 253-258.

Brosnan, S. F., Silk, J., Henrich, J., Mareno, M. C., Lambeth, S. P. \& Schapiro, S. J 2009. Chimpanzees (Pan troglodytes) do not develop contingent reciprocity in an experimental task. Animal Cognition, 12, 587-597.

Brosnan, S. F., Houser, D., Xiao, E., Leimgruber, K., Chen, T. \& de Waal, F. B. M 2010. Competing demands of prosociality and equity in monkeys. Evolution and Human Behavior, 31, 279-288.

Burkart, J. M., Fehr, E., Efferson, C. \& van Schaik, C. P. 2007. Other-regarding preferences in a non-human primate: common marmosets provision food altruistically. Proceedings of the National Academy of Sciences, U.S.A., 104, 1976219766.

Chang, S. W. C., Winecoff, A. A. \& Platt, M. L. 2011. Vicarious reinforcement in rhesus macaques (Macaca mulatta). Frontiers in Neuroscience, 5, 27.

Chang, S. W. C., Barter, J. W., Ebitz, R. B., Watson, K. K. \& Platt, M. L. 2012. Inhaled oxytocin amplifies both vicarious reinforcement and self reinforcement in rhesus macaques (Macaca mulatta). Proceedings of the National Academy of Sciences, U.S.A., 109, 959-964.

Colman, A. D., Liebold, K. E. \& Boren, J. J. 1969. A method for studying altruism in monkeys. The Psychological Record, 19, 401-405.

Costin, S. E. \& Jones, D. C. 1992. Friendship as a facilitator of emotional responsiveness and prosocial interventions among young children. Developmenta Psychology, 28, 941-947.

Cronin, K. A., Schroeder, K. K. E., Rothwell, E. S., Silk, J. B. \& Snowdon, C. T. 2009 Cottontop tamarins do not provide rewards to their long-term mates. Journal of Comparative Psychology, 123, 231-241.

Cronin, K. A., Schroeder, K. K. E. \& Snowdon, C. T. 2010. Prosocial behaviour emerges independent of reciprocity in cottontop tamarins. Proceedings of the Royal Society B, 277, 3845-3851.

Dufour, V., Pelé, M., Neumann, M., Thierry, B. \& Call, J. 2009. Calculated reciprocity after all: computation behind token transfers in orang-utans. Biology Letters, 5, 172-175.

Eisenberg, N., Fabes, R. A. \& Spinrad, T. L. 2006. Prosocial development. In Handbook of Child Psychology (Ed. by N. Eisenberg), pp. 646-718. New York: J. Wiley.

Fehr, E. \& Fischbacher, U. 2003. The nature of human altruism. Nature, 435, 785791.

Fehr, E., Bernhard, H. \& Rockenbach, B. 2008. Egalitarianism in young children Nature, 458, 1079-1084.

Fletcher, G. E. 2008. Attending to the outcome of others: disadvantageous inequity aversion in male capuchin monkeys (Cebus apella). American Journal of Primatology, 70, 901-905.

Gilby, I. C. 2006. Meat sharing among the Gombe chimpanzees: harassment and reciprocal exchange. Animal Behaviour, 71, 953-963.

Hare, B., Melis, A. P., Woods, V., Hastings, S. \& Wrangham, R. 2007. Tolerance allows bonobos to outperform chimpanzees on a cooperative task. Current Biology, 17, 619-623.

Hay, D. F., Castle, J., Davies, L., Demetriou, H. \& Stimson, C. A. 1999. Prosocial action in very early childhood. Journal of Child Psychology and Psychiatry, 40, 905-916.

Hirata, S. 2007. Competitive and cooperative aspects of social intelligence in chimpanzees. Japanese Journal of Animal Psychology, 57, 29-40.

Horner, V., Carter, J. D., Suchak, M. \& de Waal, F. B. M. 2011. Spontaneous prosocial choice by chimpanzees. Proceedings of the National Academy of Sciences, U.S.A. 108, 13847-13851.

Jaeggi, A. V., Burkart, J. M. \& van Schaik, C. P. 2010. On the psychology of cooperation in humans and other primates: combining the natural history and experimental evidence of prosociality. Philosophical Transactions of the Roya Society B, 365, 2723-2735.

Jensen, K., Hare, B., Call, J. \& Tomasello, M. 2006. What's in it for me? Self-regard precludes altruism and spite in chimpanzees. Proceedings of the Royal Society $B$, 273, 1013-1021.

Lakshminarayanan, V. R. \& Santos, L. R. 2008. Capuchin monkeys are sensitive to others' welfare. Current Biology, 18, R999-R1000.

Massen, J., van den Berg, L., Spruijt, B. \& Sterck, E. H. M. 2010. Generous leaders and selfish underdogs: pro-sociality in despotic macaques. PLOS ONE, $\mathbf{5}$ e9734.

Massen, J., Luyten, I., Spruijt, B. \& Sterck, E. 2011. Benefiting friends or dominants: prosocial choices mainly depend on rank position in long-tailed macaques (Macaca fascicularis). Primates, 52, 237-247.

Masserman, J. H., Wechkin, S. \& Terris, W. 1964. 'Altruistic' behavior in rhesus monkeys. American Journal of Psychiatry, 121, 584-585.

Melis, A. P., Warneken, F., Jensen, K., Schneider, A., Call, J. \& Tomasello, M. 2011 Chimpanzees help conspecifics to obtain food and non-food items. Proceedings of the Royal Society B, 278, 1405-1413.

Moore, C. 2009. Fairness in children's resource allocation depends on the recipient Psychological Science, 20, 944-948.

Olson, K. R. \& Spelke, E. S. 2008. Foundations of cooperation in young children. Cognition, 108, 222-231.

Pelé, M., Dufour, V., Thierry, B. \& Call, J. 2009. Token transfers among great apes (Gorilla gorilla, Pongo pygmaeus, Pan paniscus, and Pan troglodytes): species differences, gestural requests, and reciprocal exchange. Journal of Comparative Psychology, 123, 375-384. 
Preston, S. D. \& de Waal, F. B. M. 2002. Empathy: its ultimate and proximate bases. Behavioral and Brain Sciences, 25, 1-20.

Preuschoft, S. \& van Schaik, C. P. 2000. Dominance and communication: conflict management in various social settings. In: Natural Conflict Resolution (Ed. by F. Aureli \& F. B. M. de Waal), pp. 77-105. Berkeley: University of California Press.

Rheingold, H. L., Hay, D. F. \& West, M. J. 1976. Sharing in the second year of life. Child Development, 47, 1148-1158.

Rice, G. E. J. \& Gainer, P. 1962. 'Altruism' in the albino rat. Journal of Comparative and Physiological Psychology, 55, 123-125.

Roma, P. G., Silberberg, A., Ruggiero, A. M. \& Suomi, S. J. 2006. Capuchin monkeys, inequity aversion, and the frustration effect. Journal of Comparative Psychology, 120, 67-73.

Schaub, H. 1996. Testing kin altruism in long-tailed macaques (Macaca fascicularis) in a food-sharing experiment. International Journal of Primatology, 17, 445-467.

Schwab, C., Swoboda, R., Kotrschal, K. \& Bugnyar, T. 2012. Recipients affect prosocial and altruistic choices in jackdaws, Corvus monedula. PLoS ONE, 7, e34922.

Silk, J. B. 2002. Using the 'F'-word in primatology. Behaviour, 139, 421-446.

Silk, J. B. 2008. Social preferences in primates. In: Neuroeconomics: Decision Making and the Rain (Ed. by P. W. Glimcher, C. Camerer, E. Fehr \& R. A. Poldrack), pp. 269-284. London: Elsevier.

Silk, J. B. \& House, B. R. 2011. Evolutionary foundations of human prosocial sentiments. Proceedings of the National Academy of Sciences, U.S.A., 108, 1091010917.

Silk, J. B., Brosnan, S. F., Vonk, J., Henrich, J., Povinelli, D. J., Richardson, A. S., Lambeth, S. P., Mascaro, J. \& Schapiro, S. J. 2005. Chimpanzees are indifferent to the welfare of unrelated group members. Nature, 437, 1357-1359.

Skerry, A. E., Sheskin, M. \& Santos, L. R. 2011. Capuchin monkeys are not prosocial in an instrumental helping task. Animal Cognition, 14, 647-654.

Snowdon, C. T. \& Ziegler, T. E. 2007. Growing up cooperatively: family processes and infant care in marmosets and tamarins. Journal of Developmental Processes, 2, 40-66.

Stevens, J. R. 2010. Donor payoffs and other-regarding preferences in cotton-top tamarins (Saguinus oedipus). Animal Cognition, 13, 663-670.

Svetlova, M., Nichols, S. R. \& Brownell, C. A. 2010. Toddlers' prosocial behavior: from instrumental to empathic to altruistic helping. Child Development, 81, 1814-1827.

Takimoto, A., Kuroshima, H. \& Fujita, K. 2010. Capuchin monkeys (Cebus apella) are sensitive to others' reward: an experimental analysis of food-choice for conspecifics. Animal Cognition, 13, 249-261.

Trivers, R. L. 1971. The evolution of reciprocal altruism. Quarterly Review of Biology, 46, 35-57.

Vonk, J., Brosnan, S. F., Silk, J. B., Henrich, J., Richardson, A. S., Lambeth, S. P. Schapiro, S. J. \& Povinelli, D. J. 2008. Chimpanzees do not take advantage of very low cost opportunities to deliver food to unrelated group members. Animal Behaviour, 75, 1757-1770.

de Waal, F. B. M. 2008. Putting the altruism back into altruism: the evolution of empathy. Annual Review of Psychology, 59, 279-300.

de Waal, F. B. M. \& Suchak, M. 2010. Prosocial primates: selfish and unselfish motivations. Philosophical Transactions of the Royal Society B, 365, 2711-2722.

de Waal, F. B. M., Leimgruber, K. \& Greenberg, A. R. 2008. Giving is self-rewarding for monkeys. Proceedings of the National Academy of Sciences, U.S.A., 105, 1368513689.

Warneken, F. \& Tomasello, M. 2006. Altruistic helping in human infants and young chimpanzees. Science, 311, 1301-1303.

Warneken, F. \& Tomasello, M. 2007. Helping and cooperation at 14 months of age. Infancy, 11, 271-294.

Warneken, F. \& Tomasello, M. 2009a. Varieties of altruism in children and chimpanzees. Trends in Cognitive Sciences, 13, 397-402.

Warneken, F. \& Tomasello, M. 2009b. The roots of human altruism. British Journal of Psychology, 100, 455-471.

Warneken, F., Hare, B., Melis, A. P., Hanus, D. \& Tomasello, M. 2007. Spontaneous altruism by chimpanzees and young children. PLoS Biology, 5, 1414-1420.

Westergaard, G. C., Evans, T. A. \& Howell, S. 2007. Token mediated tool exchange between tufted capuchin monkeys (Cebus apella). Animal Cognition, 10, 407414.

Wobber, V., Hare, B., Maboto, J., Lipson, S., Wrangham, R. \& Ellison, P. T. 2010 Differential changes in steroid hormones before competition in bonobos and chimpanzees. Proceedings of the National Academy of Sciences, U.S.A., 107, 12457-12462.

Wolfle, D. L. \& Wolfle, H. M. 1939. The development of cooperative behavior in monkeys and young children. Journal of General Psychology, 55, 137-175.

Yamamoto, S. \& Tanaka, M. 2009a. How did altruism and reciprocity evolve in humans?: Perspectives from experiments on chimpanzees (Pan troglodytes). Interaction Studies, 10, 150-182.

Yamamoto, S. \& Tanaka, M. 2009b. Do chimpanzees (Pan troglodytes) spontaneously take turns in a reciprocal cooperation task? Journal of Comparative Psychology, 123, 242-249.

Yamamoto, S., Humle, T. \& Tanaka, M. 2009. Chimpanzees help each other upon request. PLOS ONE, 4, e7416.

Yamamoto, S., Humle, T. \& Tanaka, M. 2012. Chimpanzees' flexible targeted helping based on an understanding of conspecifics' goals. Proceedings of the National Academy of Sciences, U.S.A., 109, 3588-3592.

Zahavi, A. 1977. Reliability in communication systems and the evolution of altruism. In: Evolutionary Ecology (Ed. by B. Stonehouse \& C. Perrins), pp. 253-259. London: Macmillan Press. 